\title{
Subungual melanoma
}

\author{
Bijan Koushk Jalali MD, Alexander Kreuter MD
}

Cite as: CMAJ 2018 August 27;190:E1018. doi: 10.1503/cmaj.180513

A 77-year-old white woman presented to our department of dermatology with a slowly enlarging, ulcerated nodule of the nail bed of the right hallux (Figure 1). The patient reported a two-year history of discolouration and splitting of the toe nail. Recently, after the nail plate broke off spontaneously, a bleeding nodule was found. The patient had been treated previously with systemic terbinafine and ciclopirox nail lacquer for six months. She was referred by her family physician with a clinical diagnosis of trauma-induced nail injury.

On physical examination of the toe, we found a complete dystrophy of the nail plate with an ulcerated nodule (1.6 cm in diameter). Peripheral subungual melanosis was present, with extension into the proximal nail fold and beyond (Hutchinson sign). The other digits, interdigital spaces and soles were normal. No palpable popliteal or inguinal lymph nodes were present. A punch biopsy of the central nodule confirmed the diagnosis of acral melanoma (Breslow thickness of $2.5 \mathrm{~mm}$ with ulceration, and negative for $\mathrm{c}-\mathrm{Kit}$ and $B R A F$ mutations).

The lesion was removed surgically with a $2 \mathrm{~cm}$ excision margin. Staging using computed tomography and biopsy of the inguinal sentinel lymph nodes showed no extension or metastases. Initiation of adjuvant systemic therapy with nivolumab is now planned.

Subungual melanoma is an uncommon presentation of melanoma that arises from the nail matrix and may present as longitudinal melanonychia or dystrophy of the nail plate. The most important differential diagnoses of this presentation include benign acral lentiginous nevus, pyogenic granuloma or squamous cell carcinoma. Subungual melanoma is most frequently found on the thumbs and great toes. Incidence of acral melanoma is high among people of Hispanic ethnic origin and is the most common histologic type of melanoma in the black population. ${ }^{1}$

The prognosis for subungual melanoma is often poor because of the more advanced stage of presentation at first diagnosis. Black or brown pigmentation of the proximal or lateral nail folds (Hutchinson sign) is an important clinical clue for acral melanoma. ${ }^{2}$ All lesions suspicious for melanoma that cannot be excised feasibly should have either the darkest or the nodular/ulcerated portion excised. ${ }^{3}$

\section{References}

1. Wu XC, Eide MJ, King J, et al. Racial and ethnic variations in incidence and survival of cutaneous melanoma in the United States, 1999-2006. J Am Acad Dermatol 2011;65(Suppl 1):S26-37.

2. Haugh AM, Zhang B, Quan VL, et al. Distinct patterns of acral melanoma based on site and relative sun exposure. J Invest Dermatol 2018;138:384-93.

3. Desai A, Ugorji R, Khachemoune A. Acral melanoma foot lesions. Part 2: clinical presentation, diagnosis, and management. Clin Exp Dermatol 2018;43:117-23.

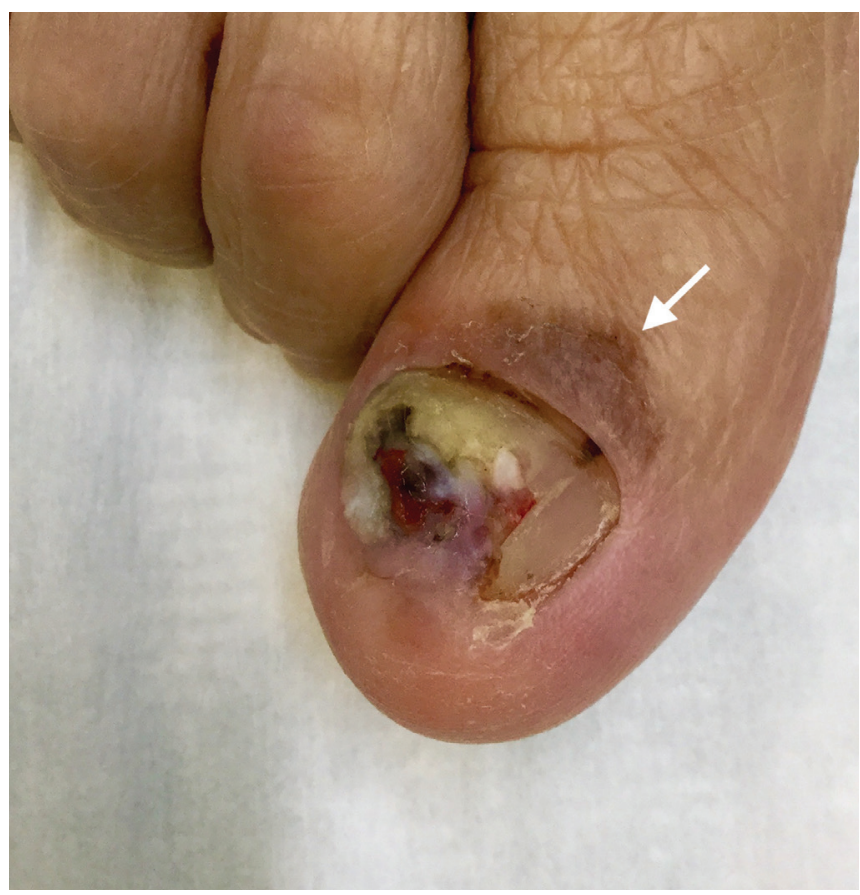

Figure 1: Right hallux of a 77-year-old woman with acral melanoma showing an ulcerated amelanotic nodule of the nail bed, complete dystrophy of the nail plate and hyperpigmentation of the proximal nail fold (arrow).

\section{Competing interests: None declared.}

This article has been peer reviewed.

The authors have obtained patient consent.

Affiliation: Department of Dermatology, Venereology, and Allergology, Helios St. Elisabeth Hospital Oberhausen, University of WittenHerdecke, Oberhausen, Germany

Correspondence to: Alexander Kreuter, a.kreuter@derma.de

Clinical images are chosen because they are particularly intriguing, classic or dramatic. Submissions of clear, appropriately labelled highresolution images must be accompanied by a figure caption. A brief explanation ( 300 words maximum) of the educational importance of the images with minimal references is required. The patient's written consent for publication must be obtained before submission. 